\title{
The Comma Sign: The Coracohumeral Ligament and Superior Glenohumeral Ligament Exhibit Similar Quantitative Characteristics With Terminal Confluence at the Subscapularis Insertion
}

\author{
Dinesh Dhanaraj, M.D., M.S.P.H., Robert L. Parisien, M.D., Kevin J. McHale, M.D., \\ Brian Cable, M.D., Antonio Cusano, M.D., Brian J. Sennett, M.D., and \\ John D. Kelly IV, M.D.
}

\begin{abstract}
Purpose: The purpose of this morphologic cadaveric study was to quantitatively define the composition of the previously described comma tissue along with its relation to the subscapularis tendon insertion. Methods: Fresh frozen cadaveric shoulder specimens were included for analysis. The coracohumeral ligament (CHL) was exposed at its origin along the base of the coracoid process and freed laterally along its course to the lesser tuberosity adjacent to the bicipital groove. The superior glenohumeral ligament (SGHL) was identified and traced along its course deep to the CHL within the rotator interval with insertion onto the superior aspect of the lesser tuberosity. The midpoint diameters of the SGHL and CHL and their composite insertional diameters on the subscapularis tendon insertion and lesser tuberosity were measured with digital calipers. The mean diameter was determined from 3 measurements taken of each ligament. Results: Eight specimens were included. With the use of digital calipers, the mean midpoint diameters of the SGHL and CHL were identified as $5.99 \mathrm{~mm}$ (range, 5.25-6.91 mm) and $5.13 \mathrm{~mm}$ (range, 4.28-5.72 mm), respectively. The composite insertional diameter of the SGHL and CHL on both the lesser tuberosity and humeral insertion of the subscapularis tendon was 9.93 $\mathrm{mm}$ (range, 6.69-12.05 mm). At its insertion, the SGHL and CHL comprised $54 \%$ and $46 \%$ of the comma tissue, respectively. Additionally, all specimens were identified as showing a confluence of the SGHL and CHL composite insertion with the subscapularis tendon at the point of its humeral head insertion. Conclusions: The comma tissue is a pivotal structure for the identification, mobilization, and repair of retracted subscapularis tendon tears. Therefore, quantitative knowledge of the midpoint diameter, insertional diameter, and composite distribution of the CHL and SGHL provided by this morphologic cadaveric analysis may aid surgeons in their efforts to restore the native anatomy. Clinical Relevance: Subscapularis tendon tears have often been under-addressed during rotator cuff repair. The comma tissue has been described as an anatomic structure that can aid in the identification, mobilization, and repair of retracted subscapularis tendon tears. Therefore, quantitative knowledge of this important arthroscopic landmark may aid surgeons in their efforts to restore the native anatomy.
\end{abstract}

I solated subscapularis tendon tears represent about $5 \%$ of rotator cuff repairs; however, when considered in conjunction with supraspinatus and infraspinatus tears, the incidence increases to $30 \%{ }^{1}$ Arthroscopic

From Penn Medicine Princeton Medical Center, Plainsboro, New Jersey, U.S.A. (D.D.); University of Pennsylvania, Philadelphia, Pennsylvania, U.S.A. (R.L.P., K.J.M., B.C., B.J.S., J.D.K.); and University of Connecticut, Farmington, Connecticut, U.S.A. (A.C.).

The authors report the following potential conflicts of interest or sources of funding: R.L.P. is on the editorial board of Arthroscopy and Arthroscopy, Sports Medicine, and Rehabilitation. J.D.K. receives book royalties from SLACK and Springer, outside the submitted work; is on the editorial board of Orthopedics; and is on the board of trustees of Arthroscopy. Full ICMJE author disclosure forms are available for this article online, as supplementary material. repair performed using a posterior viewing portal to identify the subscapularis tendon can be challenging, particularly in chronic settings in which scarring and tendon retraction may alter the native anatomy.

Received June 8, 2020; accepted December 29, 2020.

Address correspondence to Robert L. Parisien, M.D., University of Pennsylvania, 3400 Spruce St, Philadelphia, PA 19104, U.S.A. E-mail: Robert.L. Parisien@gmail.com

(C) 2020 THE AUTHORS. Published by Elsevier Inc. on behalf of the Arthroscopy Association of North America. This is an open access article under the CC BY-NC-ND license (http://creativecommons.org/licenses/by-nc-nd/4.0/). 2666-061X/20910

https://doi.org/10.1016/j.asmr.2020.12.011 
The "comma sign," originally described by Lo and Burkhart, $^{2}$ refers to a comma-shaped arc of tissue originating from the superior glenoid and the neck of the coracoid process and inserting on the superiorlateral edge of the subscapularis at its insertion on the humeral head. It consists of the coracohumeral ligament (CHL) and the superior glenohumeral ligament (SGHL), which together form the superior-medial border of the biceps sheath. ${ }^{2}$ Therefore, the comma tissue is an important landmark in identifying the superior and lateral margins of the torn subscapularis tendon, particularly in complete chronic tears with significant medial retraction and scarring to the deltoid fascia. Specifically, as the torn upper border of the subscapularis tendon retracts medially, this superiorlateral bundle of fibers forms a gentle comma-shaped curve in conjunction with the tendon. Proper arthroscopic identification of the comma sign and mobilization of the comma tissue are required to re-create the anatomic insertion of the superior-lateral border of the subscapularis tendon to the lesser tuberosity, thus optimizing patient outcomes.

The bony insertions of the rotator interval (RI) ligaments and rotator cuff tendons have been well described in the literature. However, their direction and shape are not as well understood. Anatomic studies have sought to delineate these relations and their interaction with nearby structures such as the articular capsule and subscapularis tendons. ${ }^{1,3}$ Nonetheless, quantitative morphologic and anatomic assessments of the CHL and SGHL are limited. Additionally, the distinct contributions of the SGHL and CHL to the comma tissue have yet to be determined. The purpose of this morphologic cadaveric study was to quantitatively define the composition of the previously described comma tissue along with its relation to the subscapularis tendon insertion. We hypothesized a quantitatively equal distribution of both the CHL and SGHL to the comma tissue with integration into the subscapularis tendon at its point of insertion on the lesser tuberosity.

\section{Methods}

Approval of the Institution Operational Committee for the Use of Cadavers and Recognizable Body Parts was obtained. Fresh frozen cadaveric shoulder specimens were included for analysis. Each shoulder was dissected via a wide deltopectoral approach in the simulated supine position. A combination of blunt dissection and gentle retraction was carried out to the level of the conjoint tendon. Its origin at the coracoid process was well developed to allow full visualization of its osseous structure and soft-tissue attachments. Next, the boundaries of the RI were defined at its base by the coracoid process, superiorly by the anterior margin of the supraspinatus tendon and inferiorly by the superior border of the subscapularis tendon. The dense, fibrous CHL was exposed at its origin along the base of the coracoid process and freed laterally along its course to the lesser tuberosity adjacent to the bicipital groove (Fig 1). The SGHL, originating from the superior labrum adjacent to the supraglenoid tubercle, was identified and traced along its course deep to the CHL within the RI with insertion onto the superior aspect of the lesser tuberosity (Fig 1). The CHL and SGHL were found to form a slinglike band surrounding the long head of the biceps tendon. The biceps reflection pulley, formed by the intersection of the SGHL fibers and the more medial CHL fibers, was identified. Finally, the distal aspects of the subscapularis and supraspinatus tendons were developed, and their relation with the biceps pulley was appreciated.

Once all relevant anatomic structures were appropriately visualized, the midpoint diameters of the SGHL and $\mathrm{CHL}$ and their composite insertional diameters on the subscapularis tendon insertion and lesser tuberosity were measured with digital calipers (Fig 2). The mean diameter was determined from 3 measurements taken of each ligament.

\section{Results}

Eight fresh frozen cadaveric shoulders were included for analysis. The mean age of the cadavers was 74.5 years (range, 63-92 years). All cadavers were of the white race, comprising 5 male and 3 female specimens (Table 1). Regarding the laterality of the examined specimens, 5 specimens represented the right shoulder and 3, the left. The mean cadaveric height was 168.15 $\mathrm{cm}$ (range, 154.94-175.26 cm), and the mean weight was $66.68 \mathrm{~kg}$ (range, $58.97-79.38 \mathrm{~kg}$ ).

With the use of digital calipers, the mean midpoint diameters of the SGHL and CHL were identified as 5.99 $\mathrm{mm}$ (range, 5.25-6.91 mm) and $5.13 \mathrm{~mm}$ (range, 4.28$5.72 \mathrm{~mm}$ ), respectively (Table 2). The mean composite insertional diameter of the SGHL and CHL on both the lesser tuberosity and humeral insertion of the subscapularis tendon was $9.93 \mathrm{~mm}$ (range, 6.69-12.05 $\mathrm{mm})$. At its insertion, the SGHL and CHL comprised $54 \%$ and $46 \%$ of the comma tissue, respectively. These data suggest that the comma sign is composed of a nearly equal distribution of the SGHL and CHL. Additionally, all specimens were identified as showing a confluence of the SGHL and CHL composite insertion with the subscapularis tendon at the point of its humeral head insertion.

\section{Discussion}

In this study, we found a mean midpoint diameter of $5.99 \mathrm{~mm}$ for the SGHL and $5.13 \mathrm{~mm}$ for the CHL. The composite diameter at the insertion of the comma tissue on the lesser tuberosity was $9.93 \mathrm{~mm}$. Furthermore, at the point of insertion on the lesser tuberosity and 


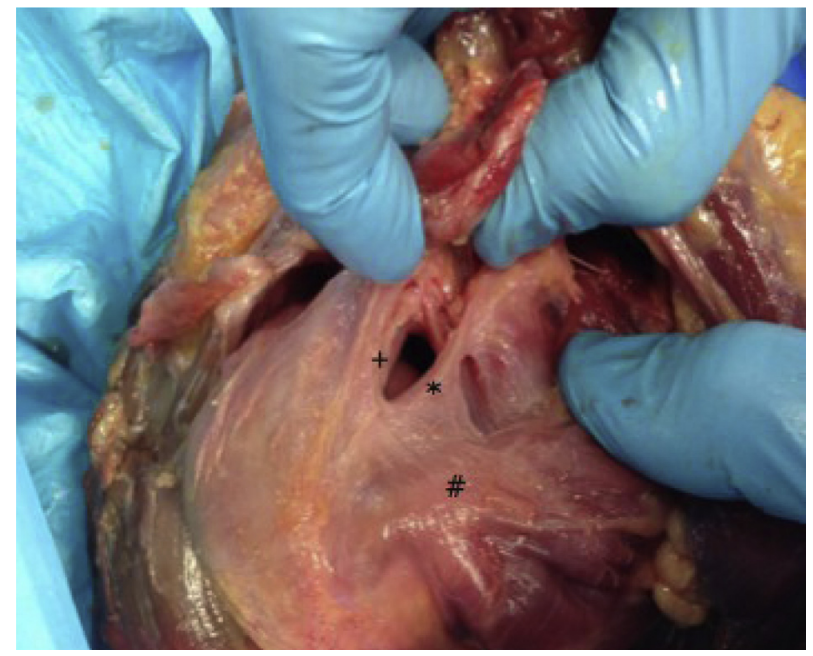

Fig 1. Gross dissection of comma tissue in right shoulder. The plus sign indicates the superior glenohumeral ligament; asterisk, coracohumeral ligament; and pound sign, subscapularis tendon.

subscapularis tendon, the comma sign consisted of $54 \%$ SGHL and $46 \%$ CHL, suggesting approximately equal contributions of the SGHL and the CHL.

Tears of the subscapularis tendon, once considered a forgotten tendon, are now readily identified arthroscopically given a modernized approach and understanding of rotator cuff pathology. Although subscapularis tendon tears have historically received less attention than those of the supraspinatus and infraspinatus tendons, the recognition and repair of subscapularis tendon tears are critically important in the restoration of the glenohumeral force couple, thus optimizing functional outcomes. ${ }^{4}$ Since its initial description by Lo and Burkhart, ${ }^{2}$ the comma sign has provided a useful intra-articular landmark in identification of retracted subscapularis tendon tears. Although much is known regarding its bony insertions, the morphologic anatomy and composition of the comma tissue require further investigation.

Several studies have reported direct non-tendinous lateral connections between the SGHL and CHL. In their cadaveric study of 23 shoulders with intact rotator cuff tendons, Clark et al. ${ }^{5}$ described the glenohumeral joint capsule as a continuous cylinder between the humerus and glenoid with the CHL located superiorly within the RI. In a follow-up anatomic and histologic analysis of 30 fresh cadavers, Clark and Harryman ${ }^{6}$ further found that the CHL and SGHL fibers were concentrated in a distinct plane between the capsule and the rotator cuff tendons. They additionally described the rotator cuff musculature as connected to the CHL and SGHL via interdigitating fibers. Gohlke et al. ${ }^{7}$ shed further light on the macroscopic anatomy and architecture of the collagen fiber bundles of the shoulder capsule in their cadaveric study of 43 shoulders. A pattern of circular and radial fibers was identified in the posterior capsule; however, a more complex network of spiral fiber bundles and complex cross-linking were found in the anterior-inferior capsule and superior capsule, respectively. This circular system contributes to the intraarticular SGHL-CHL complex and depicts the unique relation between RI structures and adjacent capsular and rotator cuff attachments.

Additional efforts have been made to better understand the specific anatomy and histology of the CHL given its biomechanical significance. Neer et al. ${ }^{8}$ studied 63 shoulder specimens and described the origin of the CHL along the lateral aspect of the coracoid. The CHL inserted into the RI in 41 of 59 shoulders and the supraspinatus tendon in 14 of 49 shoulders. A secondary slip from the ligament to the subscapularis tendon was seen in 16 shoulders. In their cadaveric analysis of 26 fresh frozen shoulder specimens, Yang et al. ${ }^{9}$ found that the CHL was located within the RI as an irregular trapezoidal structure. Its insertion site was variable, with 11 specimens inserting into the supraspinatus tendon, 11 inserting into the RI, and 1 inserting into the superior border of the subscapularis tendon. Three shoulders showed split insertions into both the supraspinatus and subscapularis tendons. Histologically, the CHL displayed similar characteristics to the joint capsule without any ligamentous features. Kolts et al. ${ }^{10}$ suggested that the CHL consisted of 2 separate segments: the inferior portion originating from the coracoid process and the coraco-glenoid ligament, which they described as representing the joint capsule anteriorly, and the pectoralis minor tendon posteriorly.

The CHL thickness has primarily been studied in the setting of adhesive capsulitis, with studies suggesting a relation between an increased CHL thickness and decreased external rotation of the glenohumeral joint. Mengiardi et al. ${ }^{11}$ compared the preoperative magnetic resonance arthrograms of 22 patients with adhesive

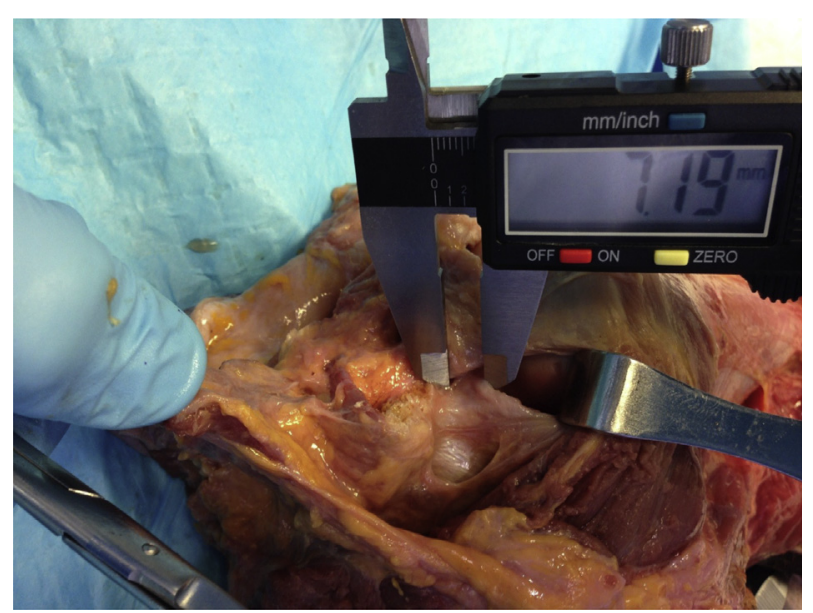

Fig 2. Quantitative assessment of comma tissue in right shoulder with digital calipers. 
Table 1. Characteristics of Cadaveric Specimens

\begin{tabular}{lc}
\hline Characteristic & Data \\
\hline Specimens, $\mathrm{n}$ & 8 \\
Laterality, n & 5 \\
$\quad$ Right & 3 \\
Left & \\
Sex, $\mathrm{n}$ & 5 \\
$\quad$ Male & 3 \\
Female & \\
Age, yr & 74.5 \\
Mean & $63-92$ \\
Range & \\
Height, cm & 168.15 \\
$\quad$ Mean & $154.94-175.26$ \\
$\quad$ Range & \\
Weight, kg & 66.68 \\
Mean & $58.97-79.38$ \\
Range &
\end{tabular}

capsulitis treated with arthroscopic capsulotomy with the magnetic resonance arthrograms of age- and sexmatched control subjects without adhesive capsulitis. They found the CHL thickness to be $2.7 \mathrm{~mm}$ in the control group and $4.1 \mathrm{~mm}$ in the frozen shoulder group. However, measuring the CHL thickness via advanced imaging has been shown to be highly user dependent as evidenced by the work of Homsi et al. ${ }^{12}$ In their analysis, they found a CHL thickness of $1.34 \mathrm{~mm}$ in asymptomatic shoulders.

Additional anatomic studies have more accurately characterized the morphology of the RI. Walch et al. ${ }^{13}$ found "hidden lesions" of the CHL, SGHL, and superior border of the subscapularis tendon in 19 of 116 cases. They described these as hidden lesions because the 19 cases were initially thought to have isolated supraspinatus tendon lesions. With open rotator cuff surgery, lesions of the RI may be easily missed if the medial border of the bicipital sheath is not noticeably disrupted. Walch et al. further found the CHL to be connected to the anterior aspect of the supraspinatus at the superiorlateral edge of the subscapularis tendon with convergence of the CHL and SGHL toward the lesser tuberosity. In an examination of 14 fresh cadaveric shoulders, Visonà et $\mathrm{al}^{3}{ }^{3}$ described a relation between the superiorlateral corner of the subscapularis tendon and a discrete bundle of fibers emanating from the CHL and supraspinatus tendon on medial traction of the detached subscapularis tendon. This cadaveric analysis supported the alternative theory of the pathoanatomy of the comma sign proposed by Dilisio and Neyton in their surgical technique article. ${ }^{14}$ Furthermore, Bennett ${ }^{15}$ described CHL connections with the SGHL, glenohumeral joint capsule, and subscapularis tendon. However, despite common connections with interdigitating fibers, this author identified distinct differences between the CHL-SGHL complex and the subscapularis tendon insertion sites. In a retrospective review of 165 consecutive arthroscopic rotator cuff and instability repairs, Bennett ${ }^{1}$ found that $47 \%$ of all subscapularis tendon tears involved the SGHL-CHL complex, further highlighting the intimate relation between RI lesions and rotator cuff pathology.

Our anatomic analysis provides further support for the confluence of the comma tissue (CHL and SGHL) with the humeral insertion of the subscapularis tendon because all 8 cadaveric specimens showed this intimate morphologic relation. Our data show that the comma sign consists of roughly equal distributions of both the SGHL and CHL at its confluent insertion to both the lesser tuberosity and subscapularis tendon, with an approximate diameter of $1 \mathrm{~cm}$. The composite morphologic distribution of these 2 structures to the comma tissue has not been previously elucidated. With the increasing advent of arthroscopic subscapularis tendon repairs, detailed knowledge of the comma tissue anatomy may aid in accurate identification of the torn superior-lateral border of the subscapularis tendon. During arthroscopic mobilization and repair, an indepth understanding of the quantitative contributions of the CHL and SGHL to the comma tissue will aid in precise anatomic repair of the leading edge of the subscapularis tendon, reconstituting the glenohumeral force couple, allowing for improved patient outcomes. Furthermore, subscapularis tendon contraction may conceivably dynamize the comma tissue, potentiating its stabilizing function. As such, additional studies are encouraged to better understand this complex relation.

\section{Limitations}

This morphologic cadaveric analysis is not without limitation. Although the comma tissue has been described as an important identifier of subscapularis retraction, our time-zero cadaveric analysis lacks clinical correlation given its inherent study design. Furthermore, quantitative analysis of the comma tissue via the use of digital calipers is inherently prone to a minor degree of human error. In addition, although we evaluated the anatomic structures previously described as comprising the comma tissue, we did not attempt to re-create the comma sign via tissue sectioning. Finally, the sample size was small.

Table 2. Quantitative Assessment of Comma Tissue

\begin{tabular}{|c|c|}
\hline Anatomic Characteristic & Data \\
\hline \multicolumn{2}{|l|}{ Midpoint diameter, mean (range), mm } \\
\hline SGHL & $5.99(5.25-6.91)^{*}$ \\
\hline CHL & $5.13(4.28-5.72)^{*}$ \\
\hline $\begin{array}{l}\text { Composite insertional diameter, mean } \\
\text { (range), mm }\end{array}$ & $9.93(6.69-12.05)^{\dagger}$ \\
\hline \multicolumn{2}{|l|}{ Contribution, \% } \\
\hline SGHL & 54 \\
\hline CHL & 46 \\
\hline
\end{tabular}




\section{Conclusions}

The comma tissue is a pivotal structure for the identification, mobilization, and repair of retracted subscapularis tendon tears. Therefore, quantitative knowledge of the midpoint diameter, insertional diameter, and composite distribution of the CHL and SGHL provided by this morphologic cadaveric analysis may aid surgeons in their efforts to restore the native anatomy.

\section{References}

1. Bennett W. Subscapularis, medial, and lateral head coracohumeral ligament insertion anatomy. Arthroscopic appearance and incidence of "hidden" rotator interval lesions. Arthroscopy 2001;17:173-180.

2. Lo IKY, Burkhart SS. The comma sign: An arthroscopic guide to the torn subscapularis tendon. Arthroscopy 2003; 19:334-337.

3. Visonà E, Cerciello S, Godenèche A, Neyton L, Fessy MH, Nové-Josserand L. The "comma sign": An anatomical investigation (dissection of the rotator interval in 14 cadaveric shoulders). Surg Radiol Anat 2015;37:793-798.

4. Burkhart SS, Brady PC. Arthroscopic subscapularis repair: Surgical tips and pearls A to Z. Arthroscopy 2006;22: 1014-1027.

5. Clark J, Sidles JA, Matsen FA. The relationship of the glenohumeral joint capsule to the rotator cuff. Clin Orthop Relat Res 1990;254:29-34.
6. Clark JM, Harryman DT II. Tendons, ligaments, and capsule of the rotator cuff. Gross and microscopic anatomy. J Bone Joint Surg Am 1992;74:713-725.

7. Gohlke F, Essigkrug B, Schmitz F. The pattern of the collagen fiber bundles of the capsule of the glenohumeral joint. J Shoulder Elbow Surg 1994;3:111-128.

8. Neer CS II, Satterlee CC, Dalskey RM, Flatow EL. The anatomy and potential effects of contracture of the coracohumeral ligament. Clin Orthop Relat Res 1992;280: 182-185.

9. Yang H-f, Tang K-l, Chen W, et al. An anatomic and histologic study of the coracohumeral ligament. J Shoulder Elbow Surg 2009; 18:305-310.

10. Kolts I, Busch LC, Tomusk H, et al. Anatomy of the coracohumeral and coracoglenoidal ligaments. Ann Anat 2000; 182:563-566.

11. Mengiardi B, Pfirrmann CWA, Gerber C. Frozen shoulder: MR arthrographic findings. Radiology 2004;233:486-492.

12. Homsi C, Bordalo-Rodrigues M, Silva JJ, Stump XMGRG. Ultrasound in adhesive capsulitis of the shoulder: Is assessment of the CHL a valuable diagnostic tool? Skeletal Radiol 2006;35:673-678.

13. Walch G, Nove-Josserand L, Levigne C, Renaud E. Tears of the supraspinatus tendon associated with "hidden" lesions of the rotator interval. J Shoulder Elbow Surg 1994;3:353-360.

14. Dilisio MF, Neyton L. Comma sign-directed repair of anterosuperior rotator cuff tears. Arthrosc Tech 2014;3: e695-e698.

15. Bennett W. Visualization of the anatomy of the rotator interval and bicipital sheath. Arthroscopy 2001;17:107-111. 